Planetary Systems in the Universe - Observation, Formation and Evolution

Proceedings IAU Symposium No. 202, (C)2004 IAU

Alan Penny, Pawel Artymowicz, Anne-Marie Lagrange, \& Sara Russell, eds.

\title{
Orbital Evolution of Planetary Systems
}

\author{
Tapan K. Chatterjee
}

Faculty of Science (FCC), Puebla A. University, Apartado Postal 1316, Puebla, Mexico. (E-mail tchat@excite.com)

\section{V.B. Magalinsky}

Dept. of Theoretical Physics, University of the Friendship of Peoples, Moscow, Russia

\begin{abstract}
It is significant that the orbits of the planets in the solar system are very nearly circular, except for Mercury and Pluto where, conceivably, due to their comparatively small sizes, the tidal forces have played a less active role. Most of the suspected planets orbiting pulsars have nearly circular orbits. These systems tend to have minimum energy and are subjected to tidal forces. We find that a planet circularizes its orbit, in an effort to attain orbital stability and the ground state. Details can be found in Magalinsky \& Chatterjee, 1997, and Magalinsky and Chatterjee, 2000.
\end{abstract}

Let $\mathbf{r} \equiv \mathbf{r}(t)$ denote the center of the mass of the protoplanet (secondary), of mass $\mathrm{m}(\ll \mathrm{M})$, with respect to its star (primary), of mass $\mathrm{M}$, considered to be a static point mass. The mass of the secondary is distributed about its center $\mathbf{r}$ ' $=0$ with density distribution $\sigma\left(\mathbf{r}^{\prime}, \mathrm{t}\right)$ and its internal movement is described by the velocity field $\mathbf{v}^{\prime}\left(\mathbf{r}^{\prime}, \mathrm{t}\right)$. During its orbital motion, it changes its size and density, due to tidal forces. Using the equation of continuity,

$$
\partial \sigma / \partial t+\sum_{\alpha=1}^{3} \partial / \partial x_{\alpha}^{\prime}\left(\sigma v_{\alpha}^{\prime}\right)=0
$$

where $\mathrm{x}_{\alpha}(\alpha=1,2,3)$ are the coordinates of the element of volume with respect to the center of mass of the secondary and

$$
\int \sigma d^{3} x^{\prime}=m=\text { constant }, \int x^{\prime} \sigma d^{3} x^{\prime}=0
$$

We assume that the secondary maintains its (shape) symmetry (in time) and the evolution of the distribution function of its density, $\sigma$, reduces to an anisotropic, variable scale transformation, with three scale factors $\lambda_{\alpha}^{\prime}(\mathrm{t})$;

$$
\sigma\left(\mathbf{r}^{\prime}, t\right)=m f(\xi) \Pi_{\alpha=1}^{3}\left[\lambda_{\alpha}^{\prime}(t)\right]^{-1}
$$

with $\xi_{\alpha}=x_{\alpha}^{\prime} / \lambda_{\alpha}^{\prime}$. Such that the internal motion of the secondary is an anisotropic change of scale (expansion or contraction). According to Equations $(2)$, the form factor, $f(\xi)$, is normalized by,

$$
\int f d^{3} \xi=1, \int \xi_{\alpha} f d^{3} \xi=0
$$


The radius of gyration of the secondary, corresponding to its moment of inertia about its axis of rotation, $\lambda=|\lambda|$, is given by,

$$
\lambda_{\alpha}=<\xi^{2}>^{1 / 2} \lambda_{\alpha}
$$

The internal velocity field of the secondary corresponding to its potential field is obtained from Equations (1) and (3) as,

$$
v_{\alpha}^{\prime}=\dot{\lambda}_{\alpha}^{\prime} \xi_{\alpha}=\left(\dot{\lambda}_{\alpha}^{\prime} / \lambda_{\alpha}^{\prime}\right) x_{\alpha}^{\prime}
$$

Thus considering the orbital motion and rotation of the secondary (but neglecting its internal thermal motion), and using the Relations (3), (4), (5) and (6), we obtain its kinetic potential energies, respectively, as,

$$
\begin{gathered}
K=(m / 2) \int\left(\dot{\mathbf{r}}^{2}+\dot{\lambda}^{2}\right) d^{3} x^{\prime} \\
\bigcup=-G M \int\left|\mathbf{r}+\mathbf{r}^{\prime}\right|^{-1} \sigma\left(\mathbf{r}^{\prime}, t\right) d^{3} \mathbf{r}^{\prime}
\end{gathered}
$$

Using Relations (3), Relation (8) can be presented in the form of a mean, as,

$$
\bigcup=-G M m<\left[\left(\mathbf{r}+\mathbf{r}^{\prime}\right)^{2}\right]^{-1 / 2}>
$$

Noting that $U$ is a convex function with respect to its argument $(\mathbf{r}+\mathbf{r}$ ' )$^{2}$, we can approximate $\bigcup$ from above by, $\bigcup_{a p} \geq \bigcup_{e x}$, i.e.,

$$
-G M m<\left[\left(\mathbf{r}+\mathbf{r}^{\prime}\right)^{2}\right]^{-1 / 2}>\leq-G M m\left[<\left(\mathbf{r}+\mathbf{r}^{\prime}\right)^{2}>\right]^{-1 / 2}
$$

where $\bigcup_{e x}$ and $\bigcup_{a p}$ denote the exact and approximate values of $\bigcup$, respectively. Taking into account Relation (5) we obtain,

$$
\bigcup_{a p}=-G M m\left(r^{2}+\lambda^{2}\right)^{-1 / 2}
$$

Now we unify the vectors, $\mathbf{r}$ and $\lambda$, in 6-dimensional space, such that,

$$
\mathbf{r} \oplus \lambda=\mathbf{R} \equiv\left(x_{1}, \ldots ., x_{6}\right)
$$

The extended space can obviously be decomposed into two (mutually) orthogonal sub-spaces: one (external) containing the positions of the centers of mass of the primary and secondary, and the other (internal) containing the scale factors corresponding to the secondary such that, in the extended space,

$$
\text { r. } \lambda=0, \quad r^{2}+\lambda^{2}=R^{2}, \quad \dot{r}^{2}+\dot{\lambda}^{2}=\dot{R}^{2}
$$

Using Equations (7), (11 ), (13) we approximate the Lagrangian of the system,

$$
L_{a p}=K-\bigcup_{a p}=(m / 2) \dot{R}^{2}+(G M m / R)
$$


The system tends to the ground state for, $\dot{R}=0$, implying $\mathrm{R}=\mathrm{R}_{0}=\Lambda / \mathrm{G} \mathrm{M}$ $=$ constant; such that we have a circular Keplerian motion in the extended space. As the system approaches its ground state, $\lambda$ approaches an insignificantly small value compared to $r$. This indicates that, as the protoplanet condenses moving in an elliptical trajectory under the influence of the gravitational force of the primary, the differential gravitational forces slowly circularize its orbit, driving the system towards its ground state. When the protoplanet has condensed into a planet, its size is very small compared to its original size and we have the limiting case of $\lambda$ tending to zero. The parametric (internal) space, representing its dimensions, can be visualized to reduce its dimensions and approach a point (the center of mass of the planet) as the same condenses and our extended space approaches the 3-dimensional space. Thus in this limit $\mathrm{R} \approx \mathrm{r} \approx \mathrm{R}_{0}$, and we expect that the planet has a nearly circular orbit in the 3-dimensional space, acquired in an effort to reach the ground state.

During the formation of the protoplanets, comets, etc., high eccentricities are favored. Even in the most favored theory of planetary formation in the protoplanetary disk by accretion of planetisimals planetesimal hypothesis, protoplanets are not sufficiently isolated from one another to be dynamically stable; agglomeration of these protoplanets into a small number of widely spaced planets requires a stage characterized by large orbital eccentricities. At the end of the growth phase the mass is almost totally contained in the protoplanets, such that their random velocities are not damped by energy equipartition with the planetesimals and mutual gravitational perturbations will induce substantial eccentricities, even if the protoplanets formed in circular orbits (e.g., Safronov, 1991). Also for a homogeneous distribution of points in phase space (representing the positions and velocities of primordial objects), we expect high eccentricities( Heggie, 1975, Huts 1986). These facts are indicative of a scenario in which the protoplanets are formed in highly elongated elliptical orbits and slowly circularize their orbits (due to interchanges between tidal and orbital energies), as they condense into planets, in an effort to attain the ground state of energy; and the evolution of planetary systems is characterized by progressive circularization of the orbits.

Acknowledgments. It is a matter of great pleasure to thank the organizers of the symposium for an excellent hospitality.

\section{References}

Heggie, D. C. 1975, MNRAS, 173, 729

Hut, P. 1985, in IAU Symp. 113, Dynamics of Star Clusters, Eds. J. Goodman, P. Hut (Dordrecht-Reidel), p. 103

Magalinsky, V.B., \& Chatterjee, T.K. 1997, Celest. Mech., 65, 399

Magalinsky, V.B., \& Chatterjee, T.K. 2000, Nuovo Cimento, in press

Safronov, V.S. 1991, Icarus, 94, 260 